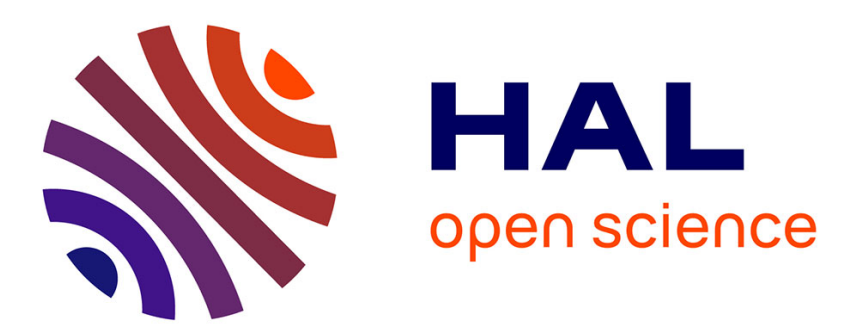

\title{
La succession de Setthāthirāt: réappréciation d'une période de l'histoire du Lān Xāng
}

\author{
Michel Lorrillard
}

\section{To cite this version:}

Michel Lorrillard. La succession de Setthāthirāt: réappréciation d'une période de l'histoire du Lān Xāng. Aséanie, Sciences humaines en Asie du Sud-Est, 1999, 4, pp.45-64. 10.3406/asean.1999.1641 . hal-02358494

\section{HAL Id: hal-02358494 https://hal.science/hal-02358494}

Submitted on 12 Nov 2019

HAL is a multi-disciplinary open access archive for the deposit and dissemination of scientific research documents, whether they are published or not. The documents may come from teaching and research institutions in France or abroad, or from public or private research centers.
L'archive ouverte pluridisciplinaire HAL, est destinée au dépôt et à la diffusion de documents scientifiques de niveau recherche, publiés ou non, émanant des établissements d'enseignement et de recherche français ou étrangers, des laboratoires publics ou privés. 


\title{
La succession de Setthāthirāt : réappréciation d'une période de l'histoire du Lān Xāng
}

In: Aséanie 4, 1999. pp. 45-64.

\begin{abstract}
The phase of unrest which spreads from the death of King Setthāthirāt (1572) to that of King Voravongsā Thammikarāt (1623) is one of the least known periods of the history of Lān Xāng - somewhat a paradox when you consider that philological and epigraphic data relative to these 50 years are more numerous than for some other periods of Laotian history.

The main purpose of this article is to establish a framework of historical events and reliable cross-checked chronological markers for this period during which the fragility of royal legitimacy turns out to be a key factor. But at the same time, comparing the available sources and assessing the insights that they offer leads the author to reflect upon problems specific to the historiography of that period and on the fact that inscriptions, although essential to historical studies, may lead to misinterpretation when not crosschecked with other sources.
\end{abstract}

Citer ce document / Cite this document :

Lorrillard Michel. La succession de Setthāthirāt : réappréciation d'une période de l'histoire du Lān Xāng. In: Aséanie 4, 1999. pp. 45-64.

doi : 10.3406/asean.1999.1641

http://www.persee.fr/web/revues/home/prescript/article/asean_0859-9009_1999_num_4_1_1641 


\title{
La succession de Setthāthirāt: réappréciation d'une période de l'histoire du Lān Xāng
}

\author{
Michel Lorrillard
}

Si certaines périodes de l'histoire du des spécialistes, il en est d'autres, comme la fin du XVI ${ }^{\mathfrak{c}}$ siècle et le début du $\mathrm{XVII}^{\mathfrak{c}}$, qui, jusqu'à présent, demeurent à peu près méconnues. Il est vrai qu'il s'agit là d'une époque que l'on a pu percevoir comme transitoire: temps troublés entre les deux règnes glorieux de Setthāthirāt (1548-1572) et de Suriyavongsā (1638-1695). Il n'empêche, c'est à peine si l'on a pu reconnaître, s'agissant de ces quelques décennies, une trame événementielle, une liste précise de souverains ou même une chronologiel. Le fait s'avère quelque peu paradoxal dans la mesure où les sources font moins défaut pour cette tranche de passé que pour d'autres. Nous avons d'abord les annales de Luang Prabang dont les plus anciennes semblent remonter au milieu du $\mathrm{XV}^{\mathrm{c}}$ siècle. Elles représentent une tradition septentrionale qui se distingue par deux types de textes: les phongsävadān et le Nithān khun borom. Ce sont eux qui nous fournissent sans conteste le plus grand nombre d'informations. Nous avons également quelques très brèves mais instructives chroniques, liées au territoire de Vientiane, qui sont probablement les fragments corrompus d'une tradition historiographique centrale commencée au $\mathrm{XVI}^{\mathfrak{c}}$ siècle et interrompue brutalement au début du XIX ${ }^{\mathfrak{c}}$. Nous possédons encore les annales des royaumes et principautés environnantes, le témoignage 
de voyageurs occidentaux, de même qu'un certain nombre de sources épigraphiques ${ }^{2}$. La recherche historique ne s'est jusqu'ici bornée qu'à l'examen d'un petit nombre de ces documents et il en résulte, plus particulièrement pour la période qui nous occupe, une vision confuse et très lacunaire du passé lao. S'il est sûr que notre connaissance du sujet demeurera toujours très superficielle, il est cependant possible, en passant par le stade de la recherche primaire - c'est-à-dire du collationnement et de l'analyse comparative - de mieux faire ressortir certains faits, de fixer des repères et de mettre en place une chronologie plus sûre. Nous nous intéresserons ici aux règnes qui suivirent celui de Setthāthirāt jusqu'à 1623, date présumée de la mort de Voravongsā Thammikarāt.

Alors que les textes lao relatent d'une façon relativement prolixe les événements marquants du règne de Setthāthirāt, ils demeurent étrangement discrets sur les circonstances qui, autour de 1571-72, entourèrent la mort du grand souverain. Sans autrement l'expliquer, on parle de sa disparition à la suite d'une campagne qu'il aurait menée contre un territoire du Sud appelé Muang Ongkān. Certains ont voulu reconnaître dans ce nom la région actuelle d'Attopeu; d'autres, s'appuyant sur la ressemblance phonétique du terme avec celui d'Angkor, y ont vu tout simplement une référence au pays khmer. En fait, il semble bien, au regard des chroniques cambodgiennes, que ce soit cette dernière identification qu'il faille considérer. On y trouve en effet pour la même époque des allusions à un conflit opposant les sujets du roi Paramarājā , demeurant à Lovêk, à ceux, venus du Nord, du roi de "Srī Sattanā Gunhut", c'est-à-dire de Vientiane ${ }^{3}$. La chronologie des faits se trouve ici grossièrement vérifiée puisque si dans une inscription datant de 1567 (stèle du Vat Nong Bone, Vientiane) on fait encore référence au roi Setthāthirāt, par contre, à partir de 1572 (stèle $n^{\circ} 2$ du Vat Tham Suvanna Khuha, province de Udon Thani), c'est un personnage portant le titre de "Phra Sumangkhala Phothisat Aiyakasrarāt Sitthidet Leu Saiy [...]" qui apparaît dans l'épigraphie lao comme souverain du Lān Xāngt.

La disparition soudaine et mystérieuse de Setthāthirāt, ainsi que l'absence d'héritier en âge de lui succéder, plongèrent le royaume du Lān Xāng dans une situation de troubles que le retour des grands généraux ne fit que renforcer. Le pouvoir fut dès lors disputé entre les deux grands seigneurs en qui le défunt avait placé toute sa confiance: le Phrayā Sēn Surintha et le Phrayā Cantha Siharāt. Les versions les plus anciennes des phongsāvadā $n^{5}$ $(\mathrm{BML} 1 / 2)$ rapportent à propos du premier qu'il était né autour de 1512 et qu'il était issu d'une lignée de chefs de villages. Il est fort probable qu'il fut envoyé très jeune à Nakhon Xieng Thong (Luang Prabang) afin d'y suivre un enseignement auprès des bonzes les plus influents du royaume. 
Sa formation achevée, il quitta le froc et entra dans l'administration royale où il se fit bientôt remarquer et gravit les différents échelons. Le roi Phothisarāt (1520-1547), qui vint sans doute s'installer à Vientiane autour de 1530 AD, l'envoya occuper la fonction de gouverneur dans ce qui était peut-être sa ville d'origine: le Muang Pāk Hoay Luang. On sait d'après les textes que cette même localité, située à peu de distance au Sud-Est de Vientiane, eut dès le $\mathrm{XV}^{\mathfrak{c}}$ siècle (et peut-être même avant) une grande importance politique et économique. Lorsque le commerçant hollandais G. Van Wuysthoff y effectua une étape, quelque deux siècles plus tard, il remarqua plus spécialement qu'il s'agissait d'un "grand centre de confection de vêtements de soie et [qu']on en exportait tous les ans en grandes quantités vers le Siam, le Tonkin, le Quinam et le Cambodge ${ }^{7 "}$. Les premiers témoignages épigraphiques du Muang Pāk Hoay Luang - qui figurent d'ailleurs parmi les plus anciens de tout le territoire lao - datent de la première moitié du XVI ${ }^{\mathrm{c}}$ siècle et viennent opportunément confirmer, semble-t-il, le texte des chroniques lao. Nous avons vu ailleurs ${ }^{8}$ que la stèle $n^{\circ} 2$ du Vat Pacchantaburi (ou Vat Den Muang), datée de 1535, attestait l'historicité de l'édit promulgué par Phothisarāt afin de purifier et de fortifier l'implantation de la religion bouddhique. Il est intéressant de constater que cette même stèle, située dans ce qui est probablement le plus ancien temple de Pāk Hoay Luang, cite également, au premier rang des seigneurs présentés comme étant les plus fidèles soutiens du roi dans son œuvre de réforme religieuse, un certain Phrayā Sēn Surintharā Sai $^{9}$. Les différents noms qui furent attribués au Phrayā Sēn Surintha au cours de sa longue carrière posent quelque peu problème. Si l'on en croit le Maha Sila Viravong ${ }^{10}$, il aurait d'abord porté, tout jeune, le surnom de Chan. Les textes rapportent qu'il aurait obtenu ensuite le titre respecté de Phrayā Yot Leu Kien de même que ceux de Phrayā Sēn Surintha Leu Sai, Phrayā Sēn Surintha Khvāng Fā et Phrayā Sēn Si Meun Muang Phā, sans que l'on sache précisément dans quel ordre ces titres se succédèrent ni même s'ils n'étaient pas confondus. L'appellation "Phrayā Sēn", elle même, évoque une position militaire - ce que confirment les chroniques siamoises lorsqu'autour de 1565 elles nous citent un Phrayā Sēn Surinthara Khvāng Fā, vainqueur d'une troupe du Muang Phitsanulok ${ }^{11}$. Les prouesses du personnage paraissent nombreuses. Certaines chroniques de Luang Prabang (BML1/2) nous disent qu'il aurait été envoyé par Phothisarāt à Muang Phā Dai Nam Thā, à la frontière avec le Lān $\mathrm{Na}^{12}$. Il aurait à ce momentlà reçu le titre de Phrayā Sēn Si Meun Muang Phā, combattu les Tai-yuan et conduit à Chiang Mai le jeune prince Setthāthirāt afin qu'il y gouvernât ${ }^{13}$. La tradition du Nithān khun borom ${ }^{14}$ rapporte que quelque temps plus tard, il se serait opposé une nouvelle fois aux troupes du Lān Nā et que, victorieux, il aurait obtenu des titres de gloire. Le même texte indique que le personnage était connu sous les deux noms de Phrayā Sēn et de Phrayā Yot et qu'il était chargé du gouvernement de "Houé-Louong et de Muong Lemon". Il y a 
lieu ici de considérer tout particulièrement cette version puisque comme on le verra encore, elle fut probablement rédigée à l'instigation du Phrayā Sēn Surintha lui-même. Après 1558 , selon les plus anciens phongsāvadān, alors que Setthāthirāt renonçait définitivement au trône de Chiang Mai, il s'était vu attribuer le gouvernement de Muang Xāy (Dān Xāy), ce qui laisse supposer que le personnage avait une place prééminente lorsque le traité d'alliance avec Ayutthaya - authentifié par la stèle de Dān Xāy / Sri Song $\mathrm{Hak}^{15}$ - fut conclu. C'est dans ce même muang d'ailleurs, que le souverain lao se serait rendu au moment de la première intervention birmane (1564) afin d'y charger son dignitaire de repousser avec ses troupes les envahisseurs. Le Phrayā Sēn, devenu entre-temps sēn muang c'est-à-dire commandant suprême des armées du royaume ${ }^{16}$, devait encore, les annales siamoises nous le confirment, intervenir dans un grand nombre d'opérations dirigées contre l'ennemi birman. Il s'illustra notamment par la grande sagesse dont il fit preuve et par l'intelligence qu'il montra pour déjouer toutes les tentatives d'agression. Les liens entre Setthāthirāt et son général se renforcèrent d'autant plus que le premier prit la fille du second pour en faire sa principale épouse. De cette union naquit le prince Phra Hno Muang (ou Phra Hno Kēo Kumān), seul héritier mâle, ce qui amena naturellement le Phrayā Sēn Surintha à revendiquer la régence et la charge des affaires du royaume lorsque le souverain disparut.

La personnalité du Phrayā Sēn Surintha éclipse quelque peu celle de l'autre grand général, le Phrayā Cantha Siharāt. C'est pourtant probablement lui qui, durant le conflit avec les Birmans, avait assuré la prise de Lopburi et protégé la retraite de Setthāthirāt lorsque celui-ci avait dû fuir le tcrritoire siamois. Les phongsāvadān le présentent généralement comme étant le gouverneur de Vientiane. La chose pourrait être confirmée par l'épigraphie puisque dans une inscription sur feuille d'or datée de 1567 et trouvée au Vat Chom Tay, à Vientiane, on parle d'un certain Phrayā Siharāt Ānantha, donateur et seigneur de Vientiane ${ }^{17}$. Les annales siamoises citent son nom à de nombreuses reprises.

Toutes les chroniques laotiennes s'accordent pour relater le conflit qui très tôt éclata entre les deux hommes, chacun d'eux prétendant exercer sans partage la fonction toute puissante de régent du royaume. Les versions different cependant quant à la manière employée par le Phrayā Sēn Surintha pour venir à bout de son adversaire. Certaines, telles la tradition du Nithān khun borom ainsi qu'un texte des annales de Xieng Khouang, donnent aux faits un caractère expéditif puisqu'elles évoquent un meurtre sans aucune forme de procès ${ }^{18}$. D'autres au contraire, font état d'une médiation des bonzes qui, durant trois mois, auraient essayé de réconcilier les deux parties. Comme rien n'y faisait, un duel eut lieu, se terminant finalement par la mort du Phrayā Chantha Siharāt terrassé sur le dos de son éléphant. 
Nos sources divergent encore sur un point important entre tous: celui de la fonction exacte exercée par le Phrayā Sēn Surintha une fois que plus rien ne s'opposa à ses ambitions. S. Phinit ${ }^{19}$ a déjà révélé l'ambiguìté que présentait dans un phongsāvadān de rédaction tardive (BML3) la traduction de l'expression "kheun khong muang" puisque l'on peut aussi bien lui donner le sens de "monter sur le trône" que celui de "gouverner le pays". Le doute se trouve ici renforcé par une formule employée dans les versions plus anciennes (BML1/2) selon laquelle les dignitaires "s'entendirent à consacrer [le Phrayā Sēn Surintha] roi en remplacement du jeune prince". Le sens n'en paraît pas certain car l'on pourrait penser qu'après tout, cette fonction n'avait qu'un caractère intérimaire puisque, loin d'être niés, les droits du prince n'y étaient en fait que délégués. Cette ambiguïté se trouve balayée dans les autres versions: sans se soucier du détail, elles font du vieux dignitaire le nouveau souverain du royaume du Lān Xāng. L'événement est habituellement situé en l'année 1572. La plupart des phongsãvadän rapportent qu'il était âgé à ce moment là de soixante ans et qu'il prit dès lors le triple nom de règne de "Phra Suriya Phothisatta Ayyakarāt", de "Phra Sumangkhala" et de "Phra Cao Pū Lān" (litt. "roi grand-père-petit-fils"), ce dernier pour rappeler qu'il était le grand-père de Phra Hno Muang. Ces données sont confirmées par l'épigraphie puisque le nom royal de "Phra Cao Sumangkhala Phothisat Aiyakasrarāt Sitthidet Leu Saiy Kai Phuvanathibodi Sri Surivongsā" apparaît pour la première fois (faisant directement suite à la dernière référence à Setthāthirāt) dans une stèle de 1572 (Vat Tham Suvanna Khuha, province de Udon Thani) commémorant une offrande de terres à un temple nouvellement édifié. Le nom du souverain (dans lequel on retrouve le terme pāli ayyaka signifiant "grand-père") est étroitement associé à celui d'un enfant royal ("rājakummān", P.rājakumāaa) dont on montrera plus loin qu'il ne pouvait s'agir que du fils de Setthāthirāâ".

La situation du royaume, même après la mort du Phrayā Cantha Siharāt, n'était cependant pas redevenue calme pour autant. Le Maha Sila Viravong (dont on ne sait sur quelle source il s'appuie) rapporte que des principautés qui jusque-là étaient vassales refusèrent dès lors de se soumettre au nouveau souverain. Dans le royaume même, un certain nombre de dignitaires - fort mécontents de voir le trône occupé ainsi par un homme qui n'était pas d'ascendance royale - optèrent pour l'exil. Ils partirent jusqu'aux limites des territoires cham (!) et khmer, dans les régions de Champassak et de Roi Et, et emmenèrent avec eux nombre d'habitants de la région de Vientiane ${ }^{21}$.

C'est en petite ère 936 (1574), année kap-set ${ }^{22}$ ou 937 (1575), année rap-khai ${ }^{23}$, alors que le Phrayā Sēn Surintha était au pouvoir depuis deux ou trois ans, que nos sources situent une nouvelle invasion birmane sur le territoire du Lān Xāng. Si les textes mentionnent cet événement avec des différences tenant à la forme, ils s'avèrent en tout cas très proches quant au 
fond puisque ce qui ressort finalement, c'est cette même volonté qu'affichaient les Birmans de contrôler les affaires du Lān Xāng en plaçant sur le trône un souverain à leur dévotion. La formulation employée dans le BML3 suggère ici que le roi de Hongsavadi ${ }^{24}$ aurait agi dans le sens de la justice puisqu'ayant appris les "méfaits" du Phrayā Sēn Surintha, il "vint à la tête de son armée [pour] le capturer et inviter Phra Hno Kēo [le fils héritier de Setthāthirāt] à venir en Birmanie. En même temps, le roi birman mit sur le trône de Muang Lān Xāng Cao Phā Reua, l'uparāt et frère cadet du roi Saiya Settha qui avait été jadis emmené en captivité25". Masquant les liens de vassalité qui à ce moment là s'instaurèrent, le texte conclut avec une certaine pudeur que "depuis cette date et pendant plusieurs années, la Birmanie et le Lān Xāng eurent des relations amicales". Les phongsāvadān - s'ils ne donnent pas de raison à l'invasion birmane - s'accordent en tout cas à mentionner la capture du Phrayā Sēn Surintha et du jeune prince et leur remplacement sur le trône par le Mahā Uparāt ${ }^{26}$.

Les guillemets s'avèrent nécessaires lorsque l'on cite le successeur du Phrayā Sēn Surintha. "Mahā Uparāt", en effet, n'est pas un nom mais un titre équivalent à celui de vice-roi et l'on sera bien en peine lorsque fouillant dans les annales lao, on essayera de retrouver l'identité du personnage qu'il recouvre. Celui-ci avait été capturé avec son épouse lors de la première invasion birmane du Lān Xāng en 1564 et les textes parlaient à ce moment là soit d'un beau-frère, soit d'un frère cadet de Setthāthirāt. La logique nous amènerait plutôt à pencher vers cette seconde solution, d'autant plus que le BML3 et le Maha Sila Viravong citent respectivement les noms de Phā Reua et de Voravangso. Ces textes sont cependant des compilations tardives de sources déjà réinterprétées et l'expérience nous a montré que sur bien des faits elles se trompaient. La stèle de Dān Xāy / Sri Song Hak qui commémore en 1563 une alliance entre le royaume du Lān Xāng et celui d'Ayutthaya mentionne, aux côtés de l'uparāja siamois, un uparāja lao. Probablement s'agissait-il déjà de notre personnage. Sur ses origines, malheureusement, l'inscription ne livre aucune information.

Indépendamment de l'identité du personnage, il nous faudra porter une certaine attention à la fonction qu'il occupait avant son sacre. Il est difficile de définir d'une façon exacte les attributions de l'uparāt car cette appellation - au fil des époques et des événements - change en fait de signification. Il semble qu'elle ne soit apparue que relativement tard - à partir de la fin du XV siècle (sans doute au contact d'Ayutthaya et du Lān $\mathrm{Na}^{27}$ ) - et que par le rôle qu'on lui attribua alors, elle ait supplanté en partie la fonction de sēn muang ${ }^{28}$. À l'époque qui nous occupe, l'uparāt n'avait cependant encore probablement qu'un rôle consultatif et essentiellement honorifique. Il faudra attendre la fin du XVIII ${ }^{c}$ siècle et l'ingérence siamoise pour que, petit à petit, il devienne de façon complètement contrôlée l'héritier en puissance de la fonction royale. 
Le Mahā Uparāt aurait occupé le trône durant quatre ou cinq années. Plusieurs sources manuscrites lao racontent qu'en 1579, un homme habile et quelque peu magicien se serait fait passer pour la réincarnation de Setthāthirā $\ell^{9}$. Répandant ses paroles illuminées, il aurait abusé les populations de Muang Sok et de Muang Xieng et aurait réussi à exercer sur elles une emprise absolue. Après s'être fait bâtir un fortin à Thong Khi Khuay ${ }^{30}$, il s'était emparé de tous les petits et grands muang alentour et était ouvertement entré en conflit avec le Mahā Uparāt. Celui-ci chargea alors les Phrayā Xieng Tay, Xieng Neua et Nakhon d'aller châtier les habitants de Thong Khi Khuay mais ils se firent battre et refluèrent en désordre jusqu'à Vientiane. Les gens du Sud progressèrent quant à eux jusqu'à Muang Nakhon ${ }^{31}$ et s'y étant retranchés, ils menèrent de là des raids contre la capitale et eurent bientôt raison de sa résistance. Le Mahā Uparāt prit la fuite, décidé à rejoindre par la voie du fleuve le souverain de Hongsavadi. Il fit cependant naufrage à un endroit où l'eau était profonde et il se noya ainsi que son épouse et ses deux filles, "conformément à leur karma". Une de nos chroniques - de rédaction tardive - présente pour cette époque une double particularité puisque, si elle omet de mentionner les événements relatifs à la rébellion du Sud, elle est également la seule à rapporter une résistance que le Mahā Uparāt luimême aurait organisée contre le suzerain birman. Géographiquement, le mouvement des événements se trouve ainsi complètement inversé puisque le danger serait venu du Nord (l'expédition punitive birmane) et non du Sud (les troupes révoltées du faux Setthāthirāt) comme le rapportent la plupart des annales. Quant au Mahā Uparāt, forcé à la fuite, il aurait trouvé la mort en descendant le Mékhong - dans les rapides de Khemmarat - et non en le remontant - dans les rapides de Keng Chan. On remarquera la lacune que présentent les données épigraphiques entre 1572 et 1582 puisque durant ces neuf années intermédiaires, aucune inscription d'importance ${ }^{32}$ ne vient confirmer ou infirmer les sources manuscrites. Il est donc difficile de se prononcer d'une façon définitive sur l'historicité du règne du Mahā Uparāt.

D'après la majorité des sources, il apparaît qu'après la mort du Mahā Uparāt, le trône du Lān Xāng resta vacant durant une année. Ainsi que le rapporte le BML3, c'est le temps qu'il fallut sans doute aux Birmans pour mettre de l'ordre dans le pays. Ce n'est en effet qu'en l'année kot-si 942 de la petite ère (1580) que le souverain de Hongsavadi se décida à réinstaller sur le trône le Phrayā Sēn Surintha qu'il détenait prisonnier depuis six années. Alors que ce dernier rentrait à Vientiane - et en contre-coup peut-être de cette libéralité - une partie de la population du Lān Xāng fut déportée jusqu'au Muang Hongsavadi. Le Phrayā Phouen faisait partie des captifs mais il put s'en retourner au bout d'une vingtaine de jours. D'un âge avancé, le Phrayā Sēn Surintha nc devait ccpendant guère profiter du trône. Les sources s'accordent en effet pour le faire décéder deux ans seulement après son retour au Lān Xāng, en l'année tao-sanga 944 de la petite ère (1582). 
La chronologie ne se trouve pas contredite ici par les données épigraphiques puisque la seconde et dernière référence dans les inscriptions à "Phra Phothisat Aiyakārāt Sitthidet Leu Saiy [...]" (nom de règne du Phrayā Sēn Surintha) date de 1582 (stèle du Vat Phon Savan à Vientiane).

Le Phrayā Sēn Surintha fut remplacé sur le trône du Lān Xāng par son fils, Nakhon Noy. Il n'apparaît pas dans les sources que les Birmans aient été à l'origine de ce choix et probablement n'en furent-ils informés que tardivement. L'initiative en revint sans doute à Nakhon Noy lui-même ou peut-être - comme le mentionnent certains textes - aux dignitaires. Ce sont pourtant ces derniers ou une partie d'entre eux qui, mécontents de la façon dont régnait le nouveau souverain, allèrent au bout d'une année demander sa destitution au souverain birman. Selon certaines chroniques, en effet, Nakhon Noy "ne régnait pas avec équité" et n'observait pas les dix règles de conduite que la tradition royale impose à un souverain. Selon d'autres, c'est parce qu'il déplut aux dignitaires ou tout simplement parce que sa légitimité était contestée. Paul Le Boulanger - qui ne se prononce pas sur les raisons qui amenèrent cette destitution - y voit par contre une marque de l'affaiblissement des Birmans qui, "circonvenus par une délégation de la cour de Vientiane 33 ", ne firent qu'entériner une situation qu'ils ne pouvaient plus eux-même contrôler. Le royaume de Birmanie venait en effet de perdre son souverain-Bayinnaung - celui-là même qui, durant les trente-deux années glorieuses de son règne, avait imposé l'hégémonie birmane sur la plus grande partie de la péninsule indochinoise. Son fils et successeur - Nandabayin - n'avait ni son sens politique ni son autorité de grand chef de guerre ${ }^{34}$. Il se heurta alors "au réveil de tous les princes thais asservis; débordé par les révoltés qui grondaient de toutes parts, il ne sut pas imposer sa volonté au Lan Xang ${ }^{35 " . ~ O n ~ r e m a r q u e r a, ~}$ en effet, que si quelques-uns de nos textes rapportent la capture de Nakhon Noy par les forces birmanes, aucun d'entre eux, en revanche, ne mentionne son remplacement. Presque toutes les sources (on mettra à part les chroniques courtes de Vientiane) s'accordent au contraire à mentionner une vacance du pouvoir qui aurait duré de l'année ka-mot 945 de la petite ère (1583) à l'année 952 (1590) ou 953 (1591) soit sept ou huit ans. Si nous avons connaissance pour cette période de sept inscriptions trouvées dans la région de Vientiane, on constatera cependant qu'aucune d'entre elles ne cite un nom de roi. Ainsi que le pense Paul Le Boulanger, ce fut sans doute une période "d'absolue confusion dans le royaume laotien désormais livré aux factions dont aucune n'était capable de prendre en main le gouvernement ${ }^{36 "}$.

Toutes les sources s'accordent pour mentionner la supplique qu'auraient adressés le clergé et les dignitaires lao au souverain birman afin que soit reconduit à Vientiane Phra Hno Muang-l'unique fils héritier de Setthāthirāt - qui avait été fait prisonnier en même temps que le Phrayā Sēn Surintha. La libération fut accordée et c'est un jeune homme de vingt ans qui, après 
seize années de captivité passées en Birmanie, revint au Lān Xāng en 1591 pour y être couronné roi. Nos sources restent relativement discrètes sur ce règne. Des textes issus de différentes traditions (luang-prabannaises, phouen ou de Vientiane) rapportent pourtant qu'en l'année tao-si 955 de la petite ère (1593), le nouveau souverain alla en compagnie du Phrayā Phouen guerroyer contre des muang vassaux qui sans doute s'étaient révoltés ${ }^{37}$. La mémoire de ces faits s'est malheureusement très mal conservée. Il semble, sur la base de très vagues assertions, que le souverain d'Ayutthaya n'ait pas été étranger à l'affaire. Rien, cependant, ne permet dans les chroniques siamoises d'avoir sur ces événements plus d'informations.

Phra Hno Muang ne profita guère de son trône puisque nos sources le font habituellement décéder à l'âge de vingt-six ans, en 1596, après seulement cinq ou six années de règne. C'est sans aucun doute ce prince qui est désigné sous le nom de règne de "Vora Ratana Thamma Prasotiset [...]" dans les inscriptions des temples Sri Bun Ruang (1591), Mucchalintha Arām 1 (1594), Ho Phra Kéo (1595) et Phra Ngām Nam Mong (fin du XVI ${ }^{c}$ siècle ${ }^{38}$ ). Dans la dernière, en effet, le personnage est présenté d'une façon explicite comme étant le fils de Setthāthirāt. Or, la remarque est importante, on constate qu'en 1572 déjà, le successeur de Setthāthirāt - le Phrayā Sēn Surintha - celui-là même qui devait son élévation à sa position de régent, régnait en ayant à ses côtés un "enfant royal" (rājakumāra) qui avait pour titulature "Ratana Prasotiset [...]". Cette double mention - celle d'un souverain et d'un prince - de par son caractère unique dans les témoignages épigraphiques (il n'est pas possible d'identifier ici Nakhon Noy avec "Ratana Prasotiset" puisque d'une façon absolue, un souverain en exercice n'associe jamais à son nom son héritier naturel) portait déjà en elle-même la confirmation pour cette époque des sources manuscrites.

Comme l'a déjà relevé $S$. Phinit ${ }^{39}$, l'identité du personnage qui succède à Phra Hno Muang pose pour l'historien une réelle difficulté. Dans le BML3, par exemple, le lien de parenté entre le nouveau souverain appelé "Thammikarāt" et son prédécesseur se trouve défini d'une façon ambiguë par l'emploi de la formule "lūk nā". Si certains auteurs tel le Maha Sila Viravong et $P$. Le Boulanger lui ont, en effet, donné la signification siamoise de "fils de la sœur cadette de la mère" (cousin), on peut également - à la suite de S. Phinit - la prendre dans son acception lao et y voir un fils adoptif sans aucun lien de sang ${ }^{40}$. La confusion s'accentue lorsque l'on considère le BML1 puisque celui-ci fait de "Thammikarāt" un "lūk nā" (fils adoptif ou cousin) non pas de Phra Hno Muang mais de Setthāthirāt lui-même. Selon le même texte - rejoint par le BML2 - le nouveau souverain s'appelait d'ailleurs à ce moment là "Vongsā" et il n'aurait troqué son nom pour celui de "Phra Thammikarāt" qu'au bout de quatre années de règne, lorsque son épouse donna naissance à un fils. Or, si les deux noms de "Vongsă" et de 
"Thammikarāt" figurent bien dans le BPL, ceux-ci semblent par contre désigner deux souverains bien distincts: le premier - fils de Setthāthirāt n'aurait régné qu'un an, entre 1596 et 1597; il aurait ensuite été remplacé par le second, "lūk nā" de Phra Hno Muang. Cette présentation des choses n'apparaît cependant pas tout à fait crédible car il n'est question dans le texte ni de la mort de Vongsā, ni d'une révolution de palais, et l'on s'explique mal, donc, ce qui aurait pu motiver un tel changement.

Les sources épigraphiques présentent pour cette époque un intérêt que l'on situera à différents niveaux. Si elles permettent de vérifier l'historicité d'un souverain ayant pour nom "Phra Thammikarāt" de même que la chronologie telle qu'elle est exposée dans la plupart des manuscrits, elles confirment également l'assertion selon laquelle les deux noms de "(Vora)vongsă $\bar{a}^{41}$ " et de "Phra Thammikarāt" ne s'appliqueraient en fait qu'à un seul personnnage. Ainsi, si dans des inscriptions de 1601 (stèle du Vat Manikhot, province de Nong Khay), 1603 (stèle ${ }^{\circ}$ 44/522 du Vat Ho Phra Kéo, Vientiane), 1604 (Bouddha du Vat That Luang Tay, Vientiane) et 1615 (?) (stèle du Vat Arun, Tha Deua) seule la référence à "Phra Phothi Voravongsā (kasattāthirāt)" apparaît - elle est substituée en 1608 (stèle du Vat Sri Bun Reuang, Nong Khay) à celle de "Phra Mahā Thammika(rājathi)rāt" alors qu'en 1617 (stèle du Vat Sisattanak, Vientiane) et en 1623 (stèle du Vat Meun Man, Ban Keun), les deux noms cette fois-ci se trouvent étroitement associés pour ne plus désigner, d'une façon explicite (la mention "est appelé aussi" ne laisse aucun doute à ce sujet) qu'un seul souverain. On peut toutefois se demander, au vu d'une inscription datée de 1598 et aujourd'hui conservée au Vat Ho Phra Kéo (stèle $n^{\circ}$ 32/495) quelle est l'identité du personnage que recouvre le nom royal de "Phra V(o)rasa Ratana Sangkha Chittathi(kasa) [...]". On peut douter qu'il puisse s'agir de Phra Hno Muang (alias Phra Vora Ratana Thamma Prasothiset [...]) car l'on verrait mal pourquoi son nom se serait modifié au terme de son règne d'une façon si conséquente. S'agit-il alors de Phra Voravongsā Thammikarāt? Il est encore une autre solution: une version du Nithān-qualifiée par certains lettrés de version "Phra Voravongsā Thammikarāt" car c'est sur ce règne particulier qu'elle achève son récit - rapporte, relayée en cela par le Maha Sila Viravong ${ }^{42}$, que l'élévation sur le trône de Phra Voravongsā Thammikarāt n'avait été possible que parce que les Birmans, d'une part, l'avaient agréée et que, d'autre part, ils avaient mis une condition à leur accord: jugeant que le prince était encore jeune, ils avaient tenu à ce que son père, nommé pour la circonstance "Phra Vorapita" (litt. "excellent père") - exerçât la régence durant quelques années. La chose pourrait bien entendu tendre à confirmer l'historicité du personnage, d'autant plus que dans la "Charte en faveur du Vat Kéo"- manuscrit que Louis Finot ${ }^{43}$ date du début du XVII ${ }^{\mathfrak{e}}$ siècle - il est fait référence, dans un dernier paragraphe qui ressemble fort en fait à un colophon, à plusieurs donations qui auraient été faites en 
964 de la petite ère (1602) au Vat Kéo, alors qu'à ce moment "régnait (au Lān Xāng) Phra Vorapitathirāt Chao ${ }^{44 "}$. On verra par la suite, en fonction des événements qui devaient intervenir, que "Phra Vorapitathirāt" aurait très bien pu régner de façon effective entre 1596 et 1601 . Pourquoi ne pas identifier ce personnage, donc, avec celui qui, en 1598, apparaissait sous le nom de "Phra Virasa Ratana Sangkha Chittathikasa" ?

La version "Phra Voravongsā Thammikarāt" du Nithān khun borom et, dans une certaine mesure, celle du Maha Sila Viravong, sont également de toutcs les sources les seules à rapporter des incidents relativement graves qui auraient entraîné les troupes du Lān Xāng à combattre dans le royaume du Lān Nā. Nous avons déjà relevé que la puissance de Hongsavadi avait été fortement battue en brèche depuis la mort de Bayinnaung. Parmi les Lao qui depuis plusieurs décennies avaient été déportés en Birmanie, nombreux furent alors ceux qui virent là une occasion de s'affranchir et qui prirent la fuite. Selon la version "Phra Voravongsā Thammikarāt" qui situe ces événements en année kat-khai (c'est-à-dire en 961 de la petite ère, soit 1599), le seigneur de Chiang Mai s'interposa alors pour les renvoyer au seigneur de Hongsavadi. Certains d'entre eux auraient cependant pu arriver à destination et ils se placèrent sous la protection du "cao pho lūk" (litt. "seigneur père-enfant"; cette formule en langage commun fait référence ici à Vorapita) qui en était à ce moment-là à sa troisième année de règne. Celui-ci chargea le Phrayā Sēn Luang de partir avec des troupes et d'emmener avec lui le Phrayā Luang Muang Nān - qui depuis quelque temps était réfugié au Lān Xāng - afin de le placer sur le trône de Chiang Mai. Les muang Phrē, Nān, Nakhon, Phayao, Seung, Lo Chiang Sēn, Chiang Rai, Fāng et Hāng furent combattus et tous capitulèrent. Les troupes lao partirent ensuite encercler Chiang Mai mais il se passa longtemps sans que les défenses de la ville ne faiblissent et comme la faim commençait à faire de nombreuses victimes, la levée du siège et le retour au Lān Xāng furent décidés.

Les événements que nous rapportent les sources lao susmentionnées sont en partie confirmés par la chronique de $\mathrm{Na} \mathrm{n}^{45}$. Celle-ci raconte en effet qu'en l'année rap-mot 959 de la petite ère (il y a ici erreur sur le chiffre ear le nom de l'année correspond en réalité à 957 de la petite ère, soit 1595), Cao Cet Putra qui était Phrayā Luang Nān combattit à Pāk Ngao le Cao Fā Saravādi de Chiang Mai mais qu'il ne put le battre et fut obligé de s'enfuir au Lān Xāng. Trois années plus tard, en l'année peuk-set (1598, le texte indique à tort 962 de la petite ère), le Phrayā Luang Nān disposa de forces lao qui avaient quitté Hongsā, il leur adjoignit des forces du Muang Lān Xāng et il partit combattre le Muang Chiang Mai. Il ne put pour autant l'emporter. La nouvelle que le Phrayā Luang était revenu avec des troupes parvint cependant au Phrayā Khek qui avait été désigné par le roi de Chiang Mai pour occuper Muang Nān. Celui-ci prit peur et il partit se réfugier auprès 
de son souverain. Le Phrayā Luang Nān revint alors s'installer dans sa cité comme autrefois. Le quatrième jour de la lune décroissante du huitième mois de l'année kat-khai (1599), le Phrayā Khoy Noy captura des habitants de Nān - les Lao durent alors se retirer de Chiang Mai. En l'année roangpao (1601), le Phrayā Sēn vint pour s'emparer du Muang Nān mais il n'y réussit pas et s'enfuit. En l'année tao-yi (1602), le Phrayā Luang Nān conduisit ses troupes à l'assaut de Chiang Mai mais il fut mis en échec et s'en retourna. En l'année ka-mao (1603), le souverain de Chiang Mai-Cao Fā Saravādi - vint avec ses troupes combattre Muang Nān. La cité fut prise, son gouverneur capturé, emmené dans la capitale du Lān Nā et exécuté.

C'est sans doute cette suite d'événements que raconte par bribes la chronique de Chiang Mai: "En 960 (1598 AD), année peuk-set, les Lao en se sauvant de Hongsā vinrent [lacune] à Chiang Mai. Fuite - [lacune] allèrent au Lān Xāng [lacune] demandèrent protection à Chiang Mai cette année-là. [...] En 963 (1601 AD), année roang-pao, Ok Yama Ram Tejo fut nommé au gouvernement de Chiang Sēn. Prise du Lān Nā par les Lao du Lān Xāng, à l'exception de Phayao, Muang Fāng, Chiang Mai. Départ de Ok Yama Ram Tejo de Chiang Sēn cette année-là ${ }^{46 "}$.

On signalera également ici le témoignage du Père de Marini-daté des années 1640-1647 $\mathrm{AD}$ - selon lequel "sur la fin du siècle passé, ou vers le commencement de celui-ci [...] ce roi ou usurpateur d'Ava, dans cette conquête générale qu'il fit des royaumes de Siam et de Pegu, qu'il soumit à son obéissance, moins par le fer que le feu qu'il y mit de tous côtés, se fut aussi rendu maître de Lao, dont il écarta les véritables habitants qu'il contraignit d'aller dans le Pegu, pour le peupler; les Langiens, néanmoins, quelques années après se voyant persécutés sous une conduite si rude et si fâcheuse, et dans la dernière consternation de cet exil, et dans les mêmes sentiments et la résolution de recouvrer leur liberté, formèrent entre eux une secrète conspiration qui eut en effet tout le succès qu'ils s'en étaient proposés. Pour y réussir, ils convinrent tous qu'à jour donné ils se saoûleraient et que l'épée à la main, ils forceraient les Péguans en quelque endroit qu'ils fussent et les extermineraient entièrement. Et il est sans doute que si l?amour . de leur patrie et l'impatience de retourner chez eux n'eut pas éteint en eux la pensée de régner, ils pouvaient se rendre les maîtres du royaume et s'en conserver la possession; mais elle prévalut et après tant de beaux succès et une entreprise si hardie, ils retournèrent sous les armes en leur premier royaume de Lao, où les Péguans qui y commandaient avec insolence furent entièrement défaits et perdirent avec la vie les biens qu'ils possédaient et le royaume qu'ils avaient usurpé ${ }^{47}$...".

Si l'on en croit le Maha Sila Viravong (qui sans la citer s'appuie sur une source proche de la version "Phra Voravongsā Thammikarāt"), Phra Vorapita réserva aux troupes qui revenaient du Lān $N a \overline{~ l e ~ p l u s ~ m a u v a i s ~ a c c u e i l . ~}$ 
Prétextant leur défection devant les murs de Chiang Mai, il leur refusa en effet l'entrée à Phra Nakhon (Vientiane) et s'apprêtait même à faire exécuter leurs chefs lorsque ceux-ci - flairant sans doute le danger - se retirèrent à Sanam Phalān Sai ("l'aire de la victoire") en emmenant avec eux Phra Voravongsā qu'ils avaient gagné à leur cause. Ayant rassemblé leurs forces, ils s'attaquèrent ensuite à Phra Nakhon. Le père et le fils se combattirent ainsi durant quatre mois. Les difficultés allaient empirant, dans un camp comme dans l'autre, lorsque les bonzes s'interposèrent afin de ramener la concorde entre les deux parties. Tout le monde se réunit à Don Can et c'est là, que devant la communauté des religieux, Phra Voravongsā demanda et obtint le pardon de Phra Vorapita. Ce dernier lui remit en sus le gouvernement du royaume, ayant décidé pour lui-même d'aller s'installer avec sa famille à Nakhon Phanom. Si le Maha Sila Viravong place ces derniers événements en l'année 1603, il en est différemment avec la version Phra Voravongsā Thammikarāt qui les situe quant à elle en 1600 .

On remarquera avec intérêt que de façon succincte, la trame de ces événements s'est conservée dans certains textes d'une tradition totalement distincte: celles de la chronique courte de Vientiane ou Chothmāy het hyo vieng $\mathrm{can}^{48}$. Le texte étant bref, on donnera ici l'ensemble des informations qu'elles contiennent pour cette période: 958 (1596 AD), année roay-san, Phra Ton $\mathrm{Phi}^{49}$ meurt. Phra Vo le remplace. 961 (1599 AD), année katkhai, il y a la guerre à Phalān Sai, Phra Thammi règne. 964 (1602 AD), Phra Pita va faire la guerre à Muang Khuk. 965 (1603 AD) année ka-mao, de Muang Luang (Luang Prabang) arrivent des guerriers (?). Année roay-sanga (1606 AD), Phra Pita se fait bonze. Année peuk-san (1608 AD), sécheresse. 979 (1617 AD), Phra Pita meurt.

S'il apparaît donc tout à fait possible que Phra Vorapita ait régné de façon effective sur le Lān Xāng entre 1596 et 1599, voire 1601 (date de la première référence épigraphique directe à Phra Voravongsā Thammikarāt), il demeure fort probable, également, que celui-ci, à l'exemple de ce que suggèrent les chroniques courtes de Vientiane, ait continué à jouer un rôle après cette période. Peut-être, durant quelques années, poursuivit-il une résistance militaire contre son fils. La chose étant rendue vaine, il se peut qu'il se soit alors contenté de mener une vie pieuse et détachée des contingences politiques. En 1613, soit quelque quatre années peut-être avant sa mort, une stèle conservée au Vat Hay Sok, à Vientiane, fait mention d'un certain "Phra Vorapitathirāt Cao" en tant que donateur de terres (avec le Phrayā Rāt Nakhon Suvat) au temple nouvellement édifié. Il nous faudra par ailleurs signaler, même si celle-ci n'est malheureusement pas datée, la stèle de Bān Simano (Muang Thā Deua) où Voravongsā cite parmi les souverains qui l'ont précédé un certain "Somdet Phra Pitāhirāt" qui est manifestement son père. 
Il est étrange de constater combien les événements dont nous avons fait le récit plus haut ressemblent par leur trame à d'autres événements qui, selon la majorité des sources (y compris le Maha Sila Viravong), auraient eu lieu bien des années plus tard. En l'année roang-rao 983 de la petite ère (1621), en effet, une lutte meurtrière se serait engagée entre le souverain et son fils, mais les rôles se trouvent alors inversés puisque les belligérants, cette fois-ci, étaient Thammikarāt et son aîné: Upayuvarāt. Paul Le Boulanger présente le second comme doté d'une certaine maturité d'esprit puisque celui-ci, “à peine parvenu à l'âge d'homme, s'intéressa vivement à l'administration de son pays [...] et prit finalement une part prépondérante dans la direction des affaires du royaume ${ }^{50 "}$. Les BML1/2 indiquent quant à eux que le "père et le fils étaient ensemble souverains du Lān Xāng à Vientiane", ce qui laisse à penser que Phra Upayuvarāt avant même la mort de son père - disposait déjà de la toute-puissance royale. Selon $P$. Le Boulanger, c'est en raison de cette situation même que la discorde naquit entre les deux hommes car Thammikarāt, "blessé dans son orgueil paternel, n'admit pas cette ingérence prématurée dans ses prérogatives royales et traita son fils en rebelle ${ }^{51 "}$. Les $B M L 1 / 2$ rapportent simplement que la rupture se fit à la suite d'une calomnie et que les seigneurs se placèrent alors en bloc du côté de Phra Upayuvarāt.

Selon le Maha Sila Viravong (qui sans citer sa source donne sur cette affaire de nombreuses précisions ${ }^{52}$ ), le prince, poursuivi par les sbires de son père, aurait avec ses hommes d'abord trouvé refuge dans l'enceinte du Vat Nam Mong, puis à Vieng Khuk. C'est non loin de là, à Bān Thā Khēk où stationnait l'armée royale, que les princes et les dignitaires décidèrent soudainement tous ensemble de passer dans le camp de Phra Upayuvarāt. Quelque temps plus tard, alors qu'il s'enfuyait en direction de Bān Xieng Dēng, Phra Voravongsa Thammikarāt était rattrapé et finalement exécuté. Le Maha Sila Viravong, s'il est le seul à raconter d'une façon détaillée ces événements, se trouve rejoint par les autres sources dès qu'il s'agit du sort réservé à Phra Thammikarāt. Presque toutes s'accordent en effet à mentionner son exécution ainsi que celle de plusieurs de ses dignitaires à Xieng Dāng, en l'année tao-set de la petite ère (1622). Les données épigraphiques s'écartent ici légèrement des sources manuscrites puisque la dernière référence gravée dans la pierre à "Phra Mahā Thammikarājathirāt [...] qui porte [également] le nom de Phra Phothi Voravongsā Mahā Kasattarāthirāt Cao" date du 12 avril 1623 (stèle du Vat Meun Man, Ban Keun).

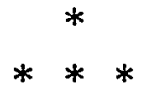


L'obscurité qui entoure l'histoire du Lān Xāng entre 1572 et 1623 s'explique d'une part par la rareté des travaux effectués sur cette période mais également par le nombre restreint des sources jusqu'à présent utilisées. Les chroniques du Laos, lorsqu'elles sont étudiées d'une façon isolée, révèlent en effet pour certaines époques de graves lacunes. Seule une analyse comparative, dans la mesure où celle-ci fait également ressortir une homogénéité d'ensemble entre les sources, permet de faire apparaître une suite d'indices qui, recoupés, redonnent à la trame historique une logique et une consistance. Les traditions annalistiques lao sont d'origines géographiques et contextuelles diverses mais se singularisent - au-delà de différences formelles imputables surtout à une certaine absence de rigueur chez les copistes - par la cohérence de leur contenu. Il n'existe pas ici de contradictions majeures; bien au contraire, dès lors que l'on s'astreint à synthétiser les données, des pans de passé s'éclairent et des suites d'événements commencent à s'enchaîner. Les sources étrangères connues - pour la plupart siamoises (en attendant une étude plus complète des chroniques birmanes et chinoises) - se recoupent ici avec les textes lao alors que les données épigraphiques, encore à peine étudiées, confirment non seulement les titulatures mais également la chronologie d'ensemble.

Lorsque l'on étudie la fin du XVI ${ }^{\mathrm{e}}$ et le début du XVII ${ }^{\mathrm{e}}$ siècle lao, on s'attachera en premier lieu à la question de la fragilité de la légitimité royale. L'accès au trône du Phrayā Sēn Surintha s'accompagne en effet d'une rupture: pour la première fois, depuis au moins Fā Ngum $^{53}$ (1353-1373 ou 74), le pouvoir tout entier passe entre les mains d'un homme d'origine roturière même si par des alliances familiales, celui-ci se trouve être le grand-père du fils héritier de Setthāthirāt. Cette entorse à la tradition ne fera en fait que s'accentuer avec l'intervention du pouvoir birman: Nakhon Noy, Phra Vorapita, Phra Voravongsā Thammikarāt (et sans doute quelques-uns de leurs successeurs directs) ne doivent en fait leur pouvoir qu'à un ensemble de circonstances (minorité du prince héritier, influence des grands seigneurs militaires, invasion étrangère, etc.) qui par la suite, même aux temps les plus sombres de l'intervention siamoise, ne se reproduiront plus ${ }^{54}$. Il y a là un changement profond qui explique peut-être directement la difficulté même que l'on a à appréhender toute cette époque. Tout se passe en effet comme si dans l'esprit des annalistes (ou des copistes) il y avait une sorte de traumatisme qui les empêchait de s'accorder sur la question essentielle des filiations. Certaines données sont occultées et faussent considérablement les schémas de succession jusqu'au règne de Suriya Vongsā inclusivement. Il y a là l'objet d'un thème de réflexion que l'on retrouve dans bien d'autres traditions annalistiques t'ai.

L'étude du passage du XVI $I^{\mathfrak{c}}$ siècle au XVII ${ }^{\mathfrak{c}}$, comparée à celle des époques qui précèdent et qui suivent, fait ressortir également un certain nombre de traits qu'il est important ici de noter. Il s'agit d'une époque tout à fait 
particulière en ce qui concerne les sources historiographiques et épigraphiques lao. Dans le cas des premières, l'historien bénéficie au départ d'un certain avantage, celui d'avoir à sa disposition un très grand nombre de traditions différentes. Aux sources luang-prabannaises - qui véhiculent des données reconnues comme historiques dès lors qu'elles abordent le début du XIV $V^{\mathrm{e}}$ siècle - il ajoute, à partir du début du XVI ${ }^{\mathrm{e}}$, les traditions annalistiques de l'ancien royaume de Vientiane. Il y a là cependant un paradoxe: la documentation, bien loin de s'étoffer, perd au contraire sa texture. Il ne subsiste des chroniques de Vientiane que quelques rares fragments qui, s'ils apportent des indices importants quant à la chronologie, s'avèrent cependant trop peu précis pour reconstruire sur leur seule base une trame événementielle. Les vieilles chroniques luang-prabannaises, quant à elles, depuis la recentralisation politique à Vientiane (second quart du $\mathrm{XVI}^{\mathrm{c}}$ siècle), semblent souffrir de ce déracinement et n'apportent plus guère que des informations éparses. La chose est significative: dès lors qu'elles retrouvent leur terreau d'origine, avec la division du royaume du Lān Xāng au début du XVIII siècle, elles s'épanouissent une nouvelle fois. Les sources épigraphiques, heureusement, permettent pour toute cette période de cimenter l'ensemble des données par ailleurs recueillies. Si le nombre et la qualité des inscriptions s'expliquent aisément en ce qui concerne le règne glorieux de Setthāthirāt, cette même richesse étonne quelque peu lorsque l'on considère la fin du $\mathrm{XVI}^{\mathrm{e}}$ siècle et le début du XVII ${ }^{\mathrm{c}}$, surtout lorsqu'elle est comparée à la production relativement pauvre des époques qui suivent. Cette continuité est en elle-même importante dans la mesure où elle masque, d'une façon sans doute voulue, la discontinuité politique et le contexte de crise qui marquent ces temps. L'élément religieux est ici essentiel: depuis au moins la fin du $\mathrm{XV}^{\mathrm{e}}$ siècle, l'idéologie du pouvoir s'est totalement transformée, faisant de l'ancien seigneur de guerre qu'était le souverain lao un monarque profondément respectueux du dharma et parfois même, reconnu comme étant cakravartin. La chose est facilement vérifiable au regard des formules dithyrambiques employées dans les inscriptions depuis le règne de Phothisarāt. Or, à partir du règne du Phrayā Sēn Surintha, on constate comme une sorte de suremploi des titulatures d'origine pāli accordées aux souverains. D'une certaine façon, donc, la pierre commémore par un curieux artifice leur légitimité. On a là précisément un exemple où les sources épigraphiques, à priori les plus sûres car matériellement les plus anciennes, donnent à la perpective historique une vision qui, sans l'apport des sources manuscrites, se trouverait nettement faussée.

Dans le dernier quart du XVI ${ }^{c}$ siècle, et ce pour la première fois dans son histoire, le Lān Xāng se trouve contrôlé par une puissance étrangère. Il ne semble pas que l'influence birmane - il est vrai extrêmement limitée dans le temps comme dans l'espace - ait laissé des marques profondes dans l'organisation socio-culturelle du royaume lao. Elle eut pourtant préalablement 
des répercussions politiques considérables, en particulier sous le règne de Setthāthirāt avec l'abandon des prétentions lao sur le royaume du Lān Nā, le déplacement de la capitale royale à Vientiane et les alliances conclues avec le royaume d'Ayutthaya. La période qui nous occupe coïncide en fait avec une apogée puis avec un affaiblissement progressif mais rapide de cette influence. L'accès au pouvoir de Nakhon Noy (le fils du Phrayā Sēn Surintha) fut sans doute reconnu à postériori par le suzerain birman. Une fois que fut libéré le dernier représentant de la légimité royale - Phra No Muang - il semble que plus rien n'ait retenu les Lao dans leur désir d'émancipation. La révolte puis la fuite des familles du Lān Xāng déplacées en Birmanie entrent dans ce contexte et marquent probablement la fin d'une dépendance qui n'était plus que théorique. Il restera encore au royaume lao de retrouver dans les décennies qui suivent une unité autour d'un pouvoir fort. Pour un temps, avec le règne de Suriya Vongsā, c'est ce qui se produira.

\section{Notes}

1. On trouvera chez Paul Levy (Histoire du Laos, Que Sais-Je? $\mathrm{n}^{\circ} 1549$, P.U.F. 1974, p. 52) une rapide présentation de la période qui s'étend entre 1571 et 1637. Paul LE BOUlanger (Histoire du Laos français, Plon, 1931) a le mérite de dresser une première chronologie, même si celle-ci, basée sur des "documents plus ou moins obscurs", lui paraît peu sûre et susceptible d'être mise en cause. Saveng PHINIT (Contribution à l'histoire du royaume de Luang Prabang, PEFEO vol. XCLI, 1987) nous livre dans son étude d'une chronique luang-prabannaise de judicieux commentaires sur cette partie de l'histoire du Lān Xāng. L'ouvrage récent de Martin STUART-FOX (The Lao Kingdom of Län Xäng: Rise and Decline, White Lotus, Bangkok, 1998) fournit une présentation synthétique et apporte quelques utiles précisions.

2. Pour la présentation de ces sources, Michel LORRILLARD (Les chroniques royales du Laos - Essai d'une chronologie des règnes des souverains lao: 1316-1887, thèse de doctorat de l'EPHE, 1995).

3. On se référera pour le détail de ces événements à l'ouvrage de Khin SoK, Chroniques royales du Cambodge ** (de 1417 à 1595 ) - Collection de textes et documents sur l'Indochine, EFEO, Paris, 1988, pp. 163-170, 175-177, 318-327, Le nom de "Sī Satanāganahuta" fut, semble-t-il, d'abord donné à Luang Prabang.

4. Pour toutes les données épigraphiques, on se reportera aux travaux de base de P.M. GAGNEUX: Contribution à la connaissance de la civilisation laotienne d'après l'épigraphie du royaume de Vientiane ( $X V^{\prime}-X I X^{e}$ siècles), thèse pour le doctorat de troisième cycle de l'EHESS, 1975 - et de Thawat PUNNOTHOK, Silä cāreuk isān (en thailandais), publication de l'Université Rama Khamheng, sans date. Les nomenclatures que nous donnons seront celles de ces deux auteurs. Le volume ${ }^{\circ} 5$ de la collection des Cāreuk nai prathet thai présente une inscription datée de 1572 (face $n^{\circ} 2$ de l'inscription $n^{\circ} 2$ du Vat Phadung Sukh) où il est vraisemblablement question du même souverain: il porte alors le nom de "Phra Aiyakothirāt Det Leu Saiy |...|". 
5. La tradition des phongsāvadān comprend des textes parfois forts différents. Par commodité, nous les distinguerons en les désignant sous des abréviations (BMLl, BML2, BML3, BPL, etc.).

6. Le Muang Pāk Hoay Luang, situé à l'embouchure du Hoay Luang avec le Mékhong, se confond avec l'actuelle localité de Phon Phisay, en territoire thailandais (province de Nong Khay).

7. J.C. LESJOSNE, Le journal de voyage de Gerrit van Wuysthoff et de ses assistants au Laos (1641-1642), p. 74, Ed. du C.D.I.L., Metz, 1993.

8. M. LORRILLARD, op. cit., p. 125.

9. T. PUNNOTHOK, op.cit., p. 237. Le nom "Surintha" provient du pāli surinda ou du sanscrit surendra et désigne le roi des dieux. Les noms d'origine indienne conservent souvent la marque du sanscrit. C'est ici le cas avec l'orthographe du nom hybride "surindra" qui, prononcé à la laotienne, deviendra "surinthara" ou "surinthon". L'allongement (arbitraire) de la voyelle finale semble indiquer que l'on favorisait à l'époque la première prononciation.

10. Cf. Phongsavadan lao, p. 73 (Edition du Ministère de l'Education, 1973).

11. On citera en particulier la version "Luang Praseuth" des annales d'Ayutthaya dont la chronologie semble plus juste. Nous nous sommes appuyés ici sur l'appareil critique de la version "Phra Chakraphat Phong" (Bangkok, 4 "édition, 1991, pp. 79-90).

12. Le lieu-dit "Phā Dai" et l'embouchure du Nam Thā sont souvent confondus. Situés un peu en aval de l'actuelle ville thaïlandaise de Chiang Khong, ils sont toujours cités dans les textes lao comme représentant la limite septentrionale du royaume du Lān Xāng.

13. La chronique de Chiang Mai recoupe les annales lao lorsqu'elle relate le couronnement de Setthāthirāt (appelé Upayyo) au Lān Nãen 1546. Elle ne cite cependant pas le Phrayā Sēn Surintha. Cf. C. NotTon, Chronique de Xieng Mai, Annales du Siam III ; P. Geuthner, Paris 1922, pp. 157-158; D.K. WYATT, A. WICHIENKEEO, The Chiang Mai Chronicle, Silkworm Books, Chiang Mai, 1995, pp. 113-114.

14. On s'appuiera ici sur une version dont il existe une traduction française par A. PAVIE: "Histoire du pays de Lan-Chang, Hom Khao (H.P.L.C.)" dans Etudes diverses II, Recherches sur l'histoire du Cambodge, du Laos et du Siam , Emest Leroux Editeurs, 1898 , p. 69.

15. Cf. Louis FINOT, "Les inscriptions du Musée de Hanoi", BEFEO, 1916, pp. 251-262.

16. Pour les attributions attenantes à cette fonction, on se reportera à notre thèse ( op. cit.).

17. P.M. GAGNEUX, op. cit., pp. 182-183.

18. On peut probablement suivre le Maha Sila Viravong ( Nithān khun borom, éd. Min. des Cultes, introduction, p. 2) lorsque celui-ci date la seconde époque de rédaction du Nithān du commencement du règne du Phrayā Sēn Surintha. Si l'on en croit en effet le colophon de la version éditée, l'historiographie de toute la période qui s'étend entre le règne de Visun et les dernières années du règne de Setthāthirāt serait imputable à un bonze - le Phra Ariya Vangso - contemporain des faits et témoin peut-être fiable des événements.

19. Op. cit., p. 220.

20. Il est fait également référence dans cette stèle au très important Mahā Sangkharāja Sumangkhala Phothiviriya Cau que l'on retrouve déjà aux côtés de Setthāthirāt en 1551 AD (stèle du Vat Phadung Sukh, province de Nong Khay) et 1567 AD (stèle du 
Vat Nong Bone, Vientiane) et qui était peut-être à l'époque le chef de la communauté bouddhique de tout le royaume, ou tout au moins de la province de Vientiane.

21. Op. cit., p. 74.

22. Cf. les versions du Chothmāy het hyo vieng can (chronique courte de Vientiane), texte $\mathrm{D}$ des annales de Xieng Khouang.

23. $C$. $\mathrm{BML}(\mathrm{s})$, texte $\mathrm{C}$ des annales de Xieng Khouang.

24. "Hongsavadi" est le terme t'ai qui désigne le royaume de Pegu.

25. S. PHINIT, op. cit., p. 58.

26. Les textes les plus importants précisent que les troupes du roi de Hongsavadi étaient constituées d'éléments man (birmans), meng (môns), ngiu (shans), yuan, pang (?) et khem (kheun de Xieng Tung).

27. Dans les chroniques royales du Laos, il ne semble pas que la mention de l' uparāt soit antérieure au troisième quart du $\mathrm{XVI}^{\mathfrak{e}}$ siècle. Les données épigraphiques (stèle de Dān Xāy / Sri Song Hak) prennent donc ici d'autant plus de relief.

28. Si la "chronique de Singhavanati" ( Notron Camille, Annales du Siam I, Paris 1926, p. 192) - texte rédigé au Lān Nā - évoque un uparāt sēn muang, il est cependant rare que les deux titres soient confondus.

29. On ne comptera pas parmi ces sources la chronique du Muang Attopeu - texte probablement daté de la fin du $\mathrm{XIX}^{\mathrm{e}}$ siècle - qui raconte d'une façon à la fois très fantaisiste et très détaillée les pseudo-aventures de Phra Say (Setthāthirāt) après sa disparition dans le Sud.

30. Thong (ou Hāt) Khi Khuay (la rizière ou la plage aux bouses de buffle) est le site sur lequel, selon la tradition, le Muang Attopeu fut bâti. Les muang Sok et Xieng (ou Xung) sont situés dans cette vaste région qui englobe aujourd'hui les territoires de Sékong et d'Attopeu.

31. Il s'agit de l'actuelle Nakhon Phanom, située sur la rive droite du Mékhong, en Thailande. Le Maha Sila Viravong l'identifie à une place beaucoup plus proche de la capitale: Vieng Khuk.

32. La stèle de Don Ron, datée de 1577 , ne livre malheureusement pas le nom de ce souverain qui offrit des terres au Vat Rājakheuhā.

33. Op. cit., p. 95.

34. Cf. G. CEDES, Les peuples de la péninsule indochinoise, Dunod, Paris 1962, p. 174; G.E. HARVEY, History of Bturma, Longmans, Green \& Co, London 1925, pp. 179-184; A.P. PHAYRE, History of Burma, Susil Gupta, London, 1867.

35. Op. cit. p. 95.

36. Ibid, p. 96.

37. Selon une version de la chronique courte de Vientiane, ainsi qu'une version du Nithān khun borom dite de "Phra Voravongsā Thammikarāt", il pourrait s'agir en particulier du Muang Ongkān, ce même muang où, deux décennies plus tôt, Setthāthirāt avait disparu.

38. Les trois premières inscriptions sont conservées au musée de Khon Ken (Thailande), la quatrième est à Vientiane au musée du Vat Ho Phra Kéo, la cinquième se trouve dans la province thailandaise de Nong Khay ( $C f$. GAGNEUX et PUNNOTHOK).

39. Op. cit. pp. 228-229. 
40. Ajoutons que la formule "lūk nā" possède un autre sens en lao, celui de "beaufils" ou de "belle-fille".

41. Le nom "Vongsā", tel qu 'il apparait souvent dans les manuscrits, semble tronqué. Il dérive du pāli vamisa (sanscrit vamiśa) et désigne la famille ou la lignée. Dans un nom, il apparaît donc toujours comme le second terme (déterminé par le premier) d'un composé. Le nom "Voravongsā" (P. vara-vamisa: noble ou excellente lignée) est probablement le nom complet.

42. Op. cit.p. 77.

43. FINOT, Louis - Recherches sur la littérature laotienne, BEFEO tome XVII, Hanoi, 1917, p. 165.

44. Ibid., p. 170.

45. Il s'agit ici d'une version manuscrite. D.K. WYATT (The Nan Chronicle, SEAP, Cornell University, 1994, pp. 65-66) et Prasœt CHURATANA, The Nan Chronicle, Data Paper Number 59, Dpt. of Asian Studies, Cornell University, 1966, pp. 24-25) présentent un récit similaire.

46. C. NOTTON, op. cit., p. 172 (nous avons harmonisé la transcription du texte avec celle que nous employons dans cet article). La version donnée par D.K. WYATT et A WICHIENKEEO, op. cit., p. 124, apparaît légèrement différente. Elle mentionne d'abord qu'en l'année 1595-96, le roi du Lān Xāng, vint pour soutenir le gouverneur de Nān dans sa prétention au trône du Lān Nā, mais que ce fut un échec et qu'il retourna au Lān Xāng. Pour 1598-99, la conclusion est différente puisqu'il est question des gens du Sud qui lancèrent une attaque contre Chiang Mai (la traduction de Notton est sans doute fautive à cet endroit).

47. Père de MARINI, Relation nouvelle et curieuse du royaume de Lao . Nous nous sommes basé ici sur le texte de l'édition originale republié dans une édition bilingue (français-lao) en 1990 (Institut Lao de Recherches en Sciences Sociales, Vientiane, pp. 367-368). Pour des raisons de police de caractères, nous avons cependant modernisé l'orthographe.

48. On utilisera ici la version du Vat Ling San qui figure parmi les sources utilisées par le Maha Sila Viravong. La "Chronique de l'Histoire du Pays de Lan-Chang", version traduite par A. Pavie (Etudes diverses II; Recherches sur l'histoire du Cambodge, du Laos et du Siam , 1898, pp. 95-102) apporte le même type d'informations.

49. “Le souverain aîné”, il s'agit manifestement de Phra Hno Muang.

50. Op. cit., p. 100.

51. Ibid.

52. Op. cit., p. 78.

53. On mettra à part le cas de Thao Khamkeut, élevé à la puissance royale par l'entremise de Nāng Mahā Thevi ( ${ }^{\mathrm{e}}$ quart du XV $\mathrm{V}^{\mathrm{e}}$ siècle). Outre les données peu vérifiables qui rendent difficile la perception de cette période, on arguera du fait que le personnage était reconnu comme la réincarnation du roi Sām Sēn Thai.

54. Au XIX ${ }^{\mathfrak{e}}$ siècle, en particulier à Luang Prabang, la fonction royale apparaissait en fait comme le grade ultime d'une hiérarchie qui était étroitement contrôlée par les Siamois. Le souverain, cependant, était toujours d'ascendance royale et possédait ainsi une vraie légitimité. 